\title{
HACIA EL LIAISON LIBRARIAN: TRANSFORMACIÓN DE SERVICIOS BIBLIOTECARIOS PARA DAR APOYO A LA DOCENCIA EN LA UNIVERSITAT OBERTA DE CATALUNYA (UOC)
}

\author{
Towards the liaison librarian: transformation of library \\ services to support teaching at the Open University of \\ Catalonia (UOC)
}

Albert Cervera-Farré, Elisabet Cervera-Biedma, Cristina López-Pérez, Gema Santos-Hermosa y Cristina Vaquer-Suñer
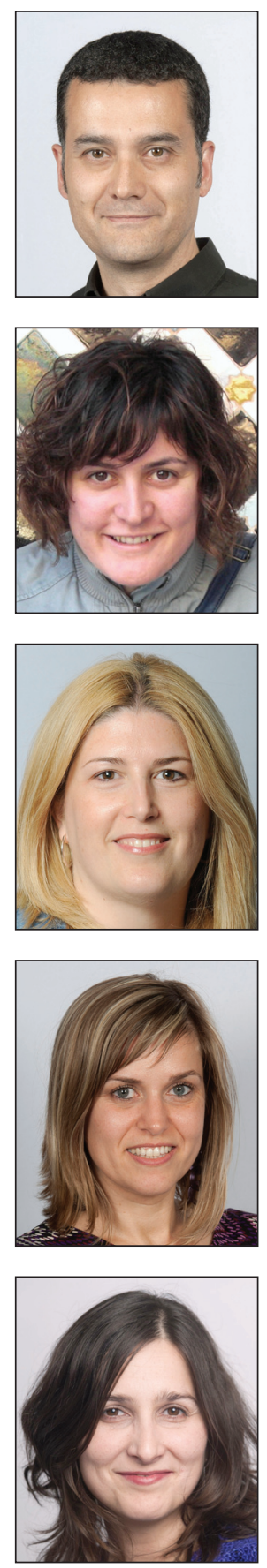

Albert Cervera-Farré es documentalista en la Biblioteca Virtual de la Universitat Oberta de Catalunya (BVUOC). Licenciado en filología por la Universitat de Barcelona (UB) y en documentación por la Universitat Oberta de Catalunya (UOC). Sus intereses se centran en la formación de usuarios en entornos virtuales y la alfabetización informacional.

http://orcid.org/0000-0002-5664-5014

acervera@uoc.edu

Elisabet Cervera-Biedma es diplomada en biblioteconomía y documentación por la Universitat de Barcelona (UB). Gestiona los recursos educativos de las aulas virtuales y ofrece apoyo documental a los estudios de psicología y educación y artes y humanidades de la Universitat Oberta de Catalunya (UOC). Especializada en libros electrónicos y lectura digital, tecnologías de la información y comunicación y medios sociales.

https://orcid.org/0000-0003-4932-9942

ecerverab@uoc.edu

Cristina López-Pérez es directora de servicios de la Biblioteca para el Aprendizaje de la Biblioteca Virtual de la UOC (BVUOC). Diplomada en biblioteconomía y documentación por la Universitat de Barcelona $(U B)$ y técnica especializada en gestión del conocimiento por la Universitat Oberta de Catalunya (UOC). Sus intereses se centran en la biblioteca universitaria, servicios y colecciones online de apoyo al estudiante y al equipo docente, y políticas y herramientas de acceso abierto.

http://orcid.org/0000-0003-4932-4084

clopezpe@uoc.edu

Gema Santos-Hermosa es licenciada en documentación por la Universitat de Barcelona (UB), master en sociedad de la información y el conocimiento por la Universitat Oberta de Catalunya (UOC). Realiza su tesis doctoral en el programa de doctorado de información y documentación en la sociedad del conocimiento de la UB. Sus intereses se centran en repositorios, recursos educativos en abierto, acceso abierto, gestión de recursos de aprendizaje, learning analytics y bibliotecas digitales.

http://orcid.org/0000-0002-2776-871X

msantoshe@uoc.edu

Cristina Vaquer-Suñer es documentalista en la Biblioteca Virtual de la Universitat Oberta de Catalunya (BVUOC). Diplomada en biblioteconomía y documentación por la Universitat de Barcelona $(U B)$ y licenciada en documentación por la Universitat Oberta de Catalunya (UOC). Especializada en acceso abierto, repositorios y recursos educativos en abierto.

http://orcid.org/0000-0002-4415-0102

mvaquer@uoc.edu

Biblioteca de la UOC Rambla del Poblenou, 156. 08018 Barcelona, España 


\title{
Resumen
}

Se describe la transformación acaecida en los últimos dos años en uno de los dos grupos operativos de la Biblioteca Virtual de la Universitat Oberta de Catalunya (UOC), el equipo de Servicios de Biblioteca para el Aprendizaje (SBA), que tiene como objetivo principal el apoyo al proceso de aprendizaje y la docencia. Se describe en detalle la elaboración de una carta de servicios específica para los docentes, cuyo propósito es acompañarles en la búsqueda y selección de recursos de aprendizaje para las asignaturas que se imparten en la UOC.

\section{Palabras clave}

Bibliotecas universitarias; Servicios bibliotecarios; Servicios de docencia; Recursos de aprendizaje; Bibliotecarios; Liaison librarians; Elearning; UOC; Biblioteca Virtual de la UOC.

\begin{abstract}
The changes over the last two years in one of the two sections of the Virtual Library, the Library Services for Learning (LSL), at the Universitat Oberta de Catalunya (UOC) are described. The main role of the $L S L$ is to provide services for the teaching and learning processes. The article describes in detail the realization of a specific services chart for the University's faculty, aimed at improving their efficiency in the identification and selection of learning resources for the disciplines taught at UOC.
\end{abstract}

\section{Keywords}

Academic libraries; Library services; Teaching and learning services, Learning resources, Librarians; Liaison librarians; Elearning; UOC; UOC Virtual Library.

Cervera-Farré, Albert; Cervera-Biedma, Elisabet; López-Pérez, Cristina; Santos-Hermosa, Gema; Vaquer-Suñer, Cristina (2015). "Hacia el liaison librarian: transformación de servicios para dar apoyo a la docencia en la Biblioteca de la UOC". El profesional de la información, v. 24, n. 2, pp. 121-129.

http://dx.doi.org/10.3145/epi.2015.mar.05

\section{Introducción}

"Save the time of the reader" fue una de las leyes que predijo Ranganathan (1931). Esta idea trasladada al caso que presentamos tiene que ver con el docente como lector o usuario, y con la forma de hacerle ahorrar tiempo, algo que pretende conseguir el servicio de apoyo a la docencia de la Biblioteca Virtual de la Universitat Oberta de Catalunya (BVUOC). Los objetivos de este servicio son dos:

- incrustar (embed) la biblioteca en los flujos de trabajo existentes (Connaway; Faniel, 2014) dentro del colectivo docente, con la finalidad de ayudarles en la preparación de la docencia; y

- participar en el diseño de las asignaturas, aportando nuestra experiencia y asesoramiento en la selección de recursos de aprendizaje para las asignaturas; garantizando su calidad e idoneidad, en línea con los contenidos tratados y actividades susceptibles de ser realizadas en las aulas y también en función de las competencias de aprendizaje incluidas en los planes docentes.

En los últimos años el rol de los profesionales de las bibliotecas universitarias está experimentando cambios importantes. Uno de ellos está relacionado con la figura del subject liaison librarian o el reference librarian y sus nuevas funciones. Éstas son entre otras: colaboración con los académicos en la elaboración de material didáctico para los cursos, formación en competencias informacionales a profesores y estudiantes, desarrollo de colecciones especializadas de apoyo a los planes docentes y asesoramiento de los docentes en los recursos de información de sus disciplinas o asignaturas (Johnson et al., 2014).

Un informe de la Association of College and Research Libraries $(A C R L, 2014)$ sugiere que los bibliotecarios debe- rían formar y asistir al personal académico en la docencia de sus cursos (en particular en lo que hace referencia a las competencias informacionales), puesto que el enfoque de "enseñar al maestro" proporciona una forma realista de llegar a más estudiantes y a más cursos. En esta línea, otras iniciativas sitúan a los bibliotecarios participando en sesiones formativas en las aulas (Gillaspy-Steinhilper, 2011; Smith; Mundt, 2014) y ofreciendo estrategias para utilizar las tecnologías y la infraestructura tecnológica (Lippincott; Vedantham; Duckett, 2014). La BVUOC pretende ampliar y actualizar estas premisas con un nuevo servicio de apoyo a la docencia, pues consideramos que la asistencia a la docencia también puede ir más allá, aprovechando nuestra experiencia profesional en la búsqueda y selección de recursos. Estas funciones de enlace con el profesorado y la incorporación a la docencia (Henry, 2012) dan lugar a la figura del liaison librarian, concepto que por otro lado no es nuevo, puesto que la literatura especializada hablaba del mismo ya en los 80s (Holbrook, 1984).

El servicio de apoyo a la docencia pretende demostrar que trabajar conjuntamente con los docentes lleva a un incremento de la calidad de los recursos de aprendizaje en las aulas virtuales

La colaboración entre bibliotecarios y profesorado es esencial para llevar a cabo estos objetivos, que requieren de la coordinación de los conocimientos de ambos colectivos. La $B V O U C$ pretende ofrecer un modelo de servicio que mejore la docencia y el aprendizaje y que a su vez demuestre que 
trabajar juntos puede llevar a un incremento de la calidad y la pertinencia de los recursos de aprendizaje en las aulas virtuales. Bajo este enfoque, al tiempo que los profesores cambian su papel de instructores a facilitadores del proceso de aprendizaje (Alam, 2010), las bibliotecas adquieren una presencia importante en las comunidades académicas de aprendizaje (Santos-Hermosa, 2012).

\section{Marco contextual: la Biblioteca Virtual de la UOC}

La BVUOC forma parte del modelo pedagógico de la UOC y es un servicio clave de apoyo al aprendizaje, la docencia, la investigación y la gestión que la Universidad pone a disposición de todos sus colectivos de usuarios.

La BVUOC nace y se desarrolla en internet desde los inicios de la propia UOC. Es por tanto un servicio entendido en y desde la virtualidad, pensado y diseñado para satisfacer las necesidades de los usuarios propios y también de quien tenga en la Red su entorno de investigación, formación y documentación académica.

\section{Los recursos de aprendizaje son uno de los tres elementos principales del mode- lo educativo de la UOC, junto con la cola- boración y el acompañamiento}

Puede accederse a la BVUOC desde el campus virtual y el portal de la UOC, pero también desde las propias aulas del campus virtual, donde tiene una presencia destacada (Cervera, 2009). De hecho, estas aulas son la principal puerta de entrada a la Biblioteca para buena parte de los estudiantes

Desde 2011 la BVUOC se organiza internamente en dos grandes equipos:

- Servicios de la Biblioteca para la Investigación (SBI). Está orientado hacia la investigación y da servicio al profesorado y otros colectivos de la Universidad en su labor investigadora.

- Servicios de la Biblioteca para el Aprendizaje (SBA). Orientado a dar servicio al proceso de aprendizaje y a la docencia. En él trabajan cinco bibliotecarios especializados en las áreas de conocimiento de la Universidad: economía y empresa; psicología y ciencias de la educación; derecho y ciencia política; informática, multimedia y telecomunicación; artes y humanidades; ciencias de la salud; y ciencias de la información y la comunicación.
La organización interna de la BVUOC y la orientación de los servicios que ofrece obedecen al nuevo modelo de biblioteca universitaria que propugna el EEES (Espacio Europeo de Educación Superior): el centro de recursos para el aprendizaje y la investigación (crai), entendido como un servicio clave en las nuevas formas de aprendizaje en la línea que describen varios autores (Domínguez-Aroca, 2005; Extremeño; Amante; Da-Costa, 2013).

\section{El origen de la transformación}

Los recursos de aprendizaje son uno de los tres elementos principales del modelo educativo de la UOC, junto con la colaboración y el acompañamiento. Éstos comprenden los contenidos, los espacios y las herramientas necesarios para llevar a cabo las actividades de aprendizaje y su evaluación.

Durante el período 2012-2013, el equipo de SBA experimentó diversas transformaciones y aglutinó servicios que en origen no coordinaba, como por ejemplo el seguimiento de la edición, la producción y el envío de los recursos de aprendizaje propios de la UOC (materiales didácticos de las asignaturas).

A finales de 2013 la estrategia (Pla estratègic 2014-2020) de edición y producción de recursos de aprendizaje de las asignaturas, así como la oferta de servicios de la biblioteca dirigidos a la docencia se vio reforzada y potenciada.

La dirección de la BVUOC vio la necesidad de iniciar un proceso de definición de una carta de servicios específica para los docentes de la Universidad, que debería ser elaborada por el equipo de SBA y en cuya definición debía contar con la participación del propio equipo docente de la Universidad e integrarse dentro de la web de la Biblioteca. La carta responde a la necesidad de poder ofrecer más eficientemente el acompañamiento al profesorado en la búsqueda y selección de recursos de aprendizaje, un elemento esencial que ofrece la BVUOC. 


\begin{tabular}{|c|c|c|}
\hline & Oportunidades & Amenazas \\
\hline \multirow[t]{2}{*}{ Externo } & $\begin{array}{l}\text { - Apoyo institucional en el rediseño de los servicios dirigidos a la } \\
\text { docencia. } \\
\text { - Creación de una nueva figura de gestión académica en la UOC, } \\
\text { el manager de programa de los estudios, cuyas funciones son } \\
\text { asegurar la viabilidad económica, marketing, comercialización, } \\
\text { gestión académica y gestión de calidad de los programas. } \\
\text { - Contexto de crisis económica. }\end{array}$ & $\begin{array}{l}\text { - Personal de gestión, y principalmente nuestros interlocutores, no } \\
\text { trabajan de la misma forma. } \\
\text { - Modelo de negocio de la editorial universitaria puede estar poco } \\
\text { alineado con algunos de los objetivos marcados por el equipo SBA } \\
\text { para la reducción de costes, como son el reaprovechamiento de } \\
\text { materiales didácticos UOC ya publicados o el uso de recursos de la } \\
\text { colección de la BVUOC. } \\
\text { - Aplicaciones de gestión desactualizadas, no responden a las nece- } \\
\text { sidades presentes. } \\
\text { - Deslocalización física de nuestros usuarios (docentes) en diversas } \\
\text { sedes. }\end{array}$ \\
\hline & Fortalezas & Debilidades \\
\hline Interno & $\begin{array}{l}\text { - Posibilidad de tener un contacto muy directo con los docentes } \\
\text { (en otras universidades no es tan fácil). } \\
\text { - Experiencia del equipo en el apoyo al profesorado y la gestión } \\
\text { de los recursos del aula. } \\
\text { - El objetivo de definición de la carta de servicios dirigida a los } \\
\text { docentes está alineado con las directrices del Plan estratégico } \\
\text { de la Universidad 2014-2020. }\end{array}$ & $\begin{array}{l}\text { - Equipo SBA no es experto en la especialización temática de las áreas } \\
\text { de conocimiento. } \\
\text { - Dependencia de otras áreas y departamentos para poder llevar a } \\
\text { cabo por completo nuestra actividad dirigida al equipo docente. }\end{array}$ \\
\hline
\end{tabular}

Figura 2. Análisis DAFO

\section{El proceso: definición de una carta de servicios para el equipo docente}

Primeramente se realizó un análisis DAFO, luego un benchmarking de casos interesantes y/o novedosos de servicios dirigidos a los docentes en activo en otras universidades, y finalmente se recogieron las opiniones de una muestra de profesores a través de una encuesta.

\subsection{DAFO}

Con el objetivo de detectar las oportunidades y amenazas externas y las fortalezas y debilidades a nivel interno, el equipo de SBA llevó a cabo un análisis DAFO. En la figura 2 se detallan los factores favorables y desfavorables que se identificaron.

También se identificaron algunas situaciones de riesgo con las que el equipo de SBA podría encontrarse (figura 3).

\begin{tabular}{|l|}
\hline Riesgos \\
\hline - Tener que ofrecer un servicio al mismo tiempo en que éste está \\
definiéndose. \\
- Exceso de personalización de los servicios. \\
- Desarrollar la oferta de servicios y que nuestros usuarios no perciban \\
su utilidad.
\end{tabular}

Figura 3. Riesgos

\subsection{Benchmarking / buenas prácticas}

Se analizaron los servicios de docencia y/o aprendizaje que ofrecían otras bibliotecas universitarias con el objetivo de encontrar buenas prácticas.

Conviene subrayar el caso de la University of Washington Instructor Toolkit como buen ejemplo de herramientas de formación dirigidas al equipo docente, especialmente las secciones de Request a customized library guide for your class y Creating stable links to journal articles. https://www.lib.washington.edu/teaching/toolkit
También se han analizado Stanford University Libraries - Research Services, University of Queensland - Teaching Support y RMIT University - Library Services for Academics and Teachers, como ejemplo en la visualización web de los servicios ofrecidos y también en la disposición de los servicios que ofrece la biblioteca en función de las necesidades del docente.

http://library.stanford.edu/research

https://www.library.uq.edu.au/_teaching-support http://www.rmit.edu.au/browse;/D=1jnvn75rp9o5

De la London School of Economics - Academics' Guide, la Queen's University Library - Teaching and Learning Support y la University of Sussex - Support for Teaching, destacan las

Tabla 1. Proporción de respuestas a la encuesta

\begin{tabular}{|l|c|c|}
\hline \multicolumn{1}{|c|}{ Áreas de conocimiento / Estudios* } & Participantes & $\%$ \\
\hline $\begin{array}{l}\text { Estudios de informática, multimedia y } \\
\text { telecomunicaciones }\end{array}$ & 27 & 26 \\
\hline Estudios de economía y empresa & 18 & 17 \\
\hline Estudios de artes y humanidades & 17 & 16 \\
\hline $\begin{array}{l}\text { Estudios de ciencias de la información y de } \\
\text { la comunicación }\end{array}$ & 16 & 15 \\
\hline Estudios de psicología y educación & 12 & 12 \\
\hline Estudios de derecho y ciencia política & 9 & 9 \\
\hline Estudios de ciencias de la salud & 2 & 2 \\
\hline Escuela de doctorado & 2 & 2 \\
\hline Sin especificar & 104 & 100 \\
\hline TOTAL
\end{tabular}

* En la UOC las facultades se denominan Estudios 
guías dirigidas a sus usuarios, así como las library toolkits. De estas últimas conviene poner especialmente de relieve la figura del liaison librarian, que tiene como finalidad asesorar en la búsqueda y la selección de los recursos para el apoyo al aprendizaje y la docencia de las asignaturas de las que los profesores son responsables. http://www.Ise.ac.uk/library/ home.aspx

http://library.queensu.ca/services/ teaching-learning-support

http://www.sussex.ac.uk/library/ teaching

En formación y/o asesoramiento sobre uso lícito de los recursos de aprendizaje en las aulas, son buenos ejemplos las miniguías de copyright localizadas en la University of Queensland - Teaching Support o la University College London - Library Services - Staff.

\subsection{Encuesta dirigida al usuario final, el docente}

Durante el período de mayo-junio de 2014 se llevó a cabo una encuesta entre la comunidad docente de la UOC con el objetivo de recoger de primera mano sus expectativas en cuanto a los servicios que necesitan que se les ofrezca desde la BVUOC para cubrir sus necesidades.

Se obtuvieron 104 respuestas de un total de 253 profesores (según datos oficiales a 31 de diciembre de 2013) en la que quedan representados los docentes de todas las áreas de conocimiento que se imparten en la UOC, en las proporciones que se muestran en la tabla 1.

La encuesta se realizó mediante un formulario de Google Forms y constaba de tres preguntas de respuesta múltiple:

a) servicios y recursos de la BVUOC que usa el profesorado habitualmente para preparar la docencia de sus asignaturas;

b) servicios y recursos de la BVUOC que el profesorado cree que serían más útiles tener disponibles en el momento de la selección de los recursos de aprendizaje de sus asignaturas;

c) momentos en los que el profesorado necesita utilizar los servicios y recursos de la BVUOC.
Las figuras 4,5 y 6 presentan en detalle los resultados obtenidos para cada pregunta en valores porcentuales.

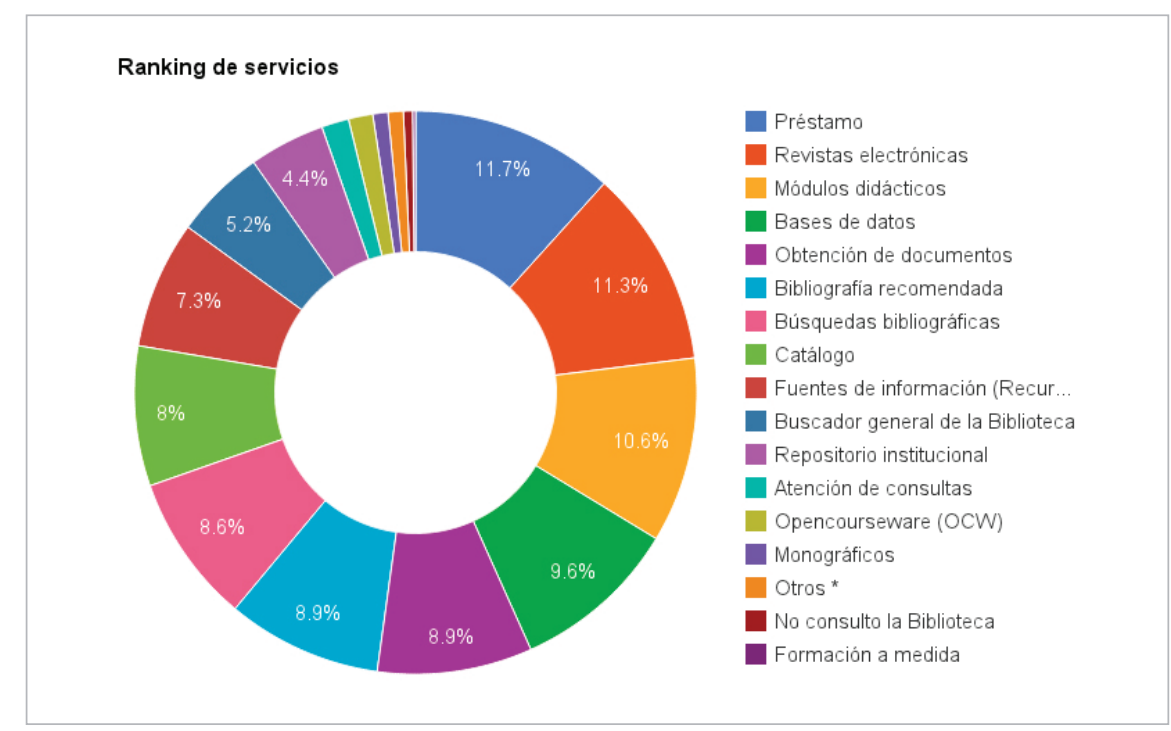

Figura 4. Servicios usados para preparar la docencia
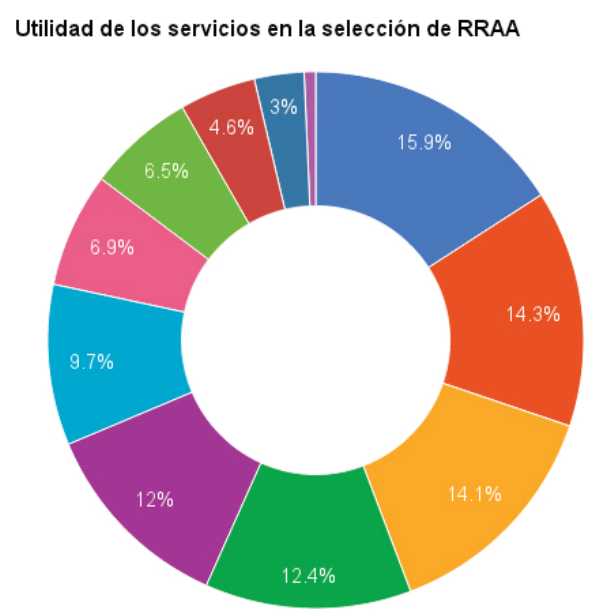

Búsqueda de recursos sobre la temática de las asignaturas

Apoyo en el reaprovechamiento de materiales de otras asignaturas Revisar y unificar la bibliografía recomendada de las asignaturas

Introducir a los estudiantes en la cita bibliográfica normalizada de 1 ...

Información sobre novedades editoriales y adquisiciones

Solicitud de dosieres monográficos sobre un tema de interés o de act

Apoyo en la evaluación de los recursos de aprendizaje (análisi d.

Apoyo a los profesores y

estudiantes en el uso y la publica...

Sesiones de formación personaliz.

Productos de formación en el uso.

口 Otros**

Figura 5. Utilidad de los servicios en el momento de la selección de los recursos de aprendizaje

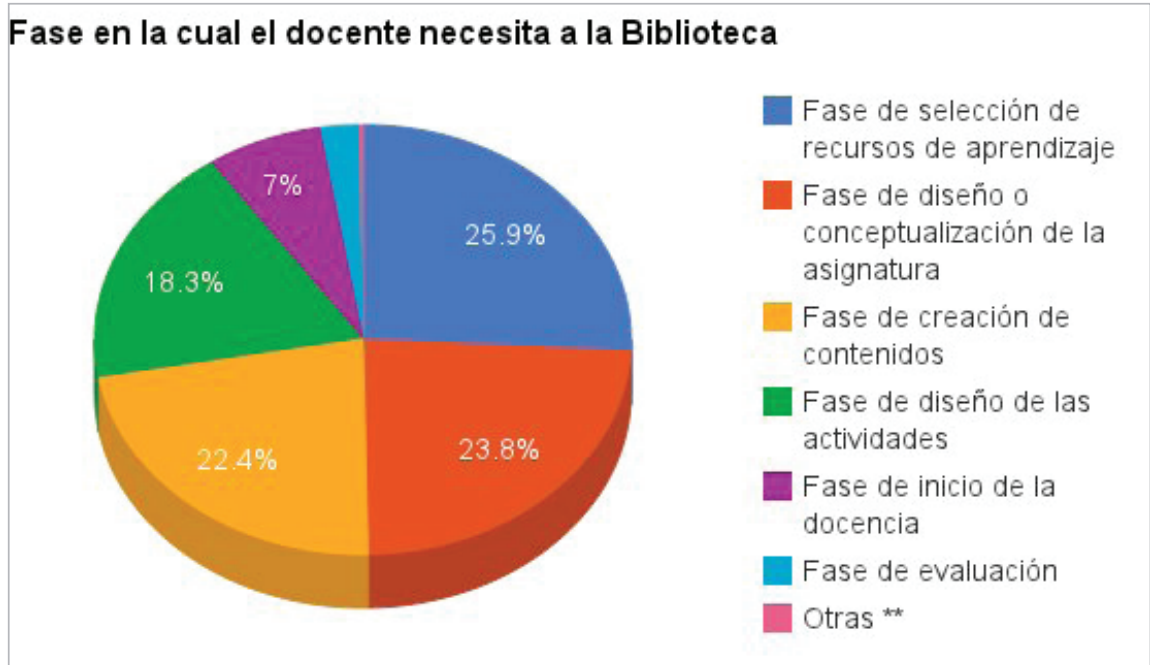

Figura 6. Fases en las que el docente necesita la Biblioteca 


\subsection{Misión, visión y objetivos}

Una vez finalizado el ejercicio de autoanálisis interno mediante el estudio DAFO, la búsqueda de buenas prácticas y la consulta de las expectativas de los docentes de la Universidad a través de la encuesta, el siguiente paso fue trabajar la definición de la misión y la visión como equipo de los servicios bibliotecarios dirigidos al aprendizaje y la docencia.

Para llevarla a cabo, el equipo de SBA consensuó como misión principal: el apoyo al profesorado y el acompañamiento en la selección de los recursos de aprendizaje para las asignaturas que imparten en la Universidad, la rentabilización del uso de la colección de la Biblioteca, haciéndola crecer adaptándose a las necesidades docentes y garantizando la sostenibilidad económica y el cuidado en el uso ético de la información.

Los objetivos de SBA son asesorar y formar a los docentes sobre las fuentes de información disponibles y la búsqueda de recursos de aprendizaje adecuados para su docencia

Por otro lado, también marcó su deseo a largo plazo, es decir, a qué aspira como equipo responsable de servicios bibliotecarios dirigidos a la docencia. La decisión unánime fue: convertirse en el referente para toda la comunidad universitaria como especialistas en recursos de aprendizaje y apoyo documental a los profesores.

Partiendo de la misión y visión expuesta anteriormente, se enumeraron los principales objetivos que el equipo de SBA

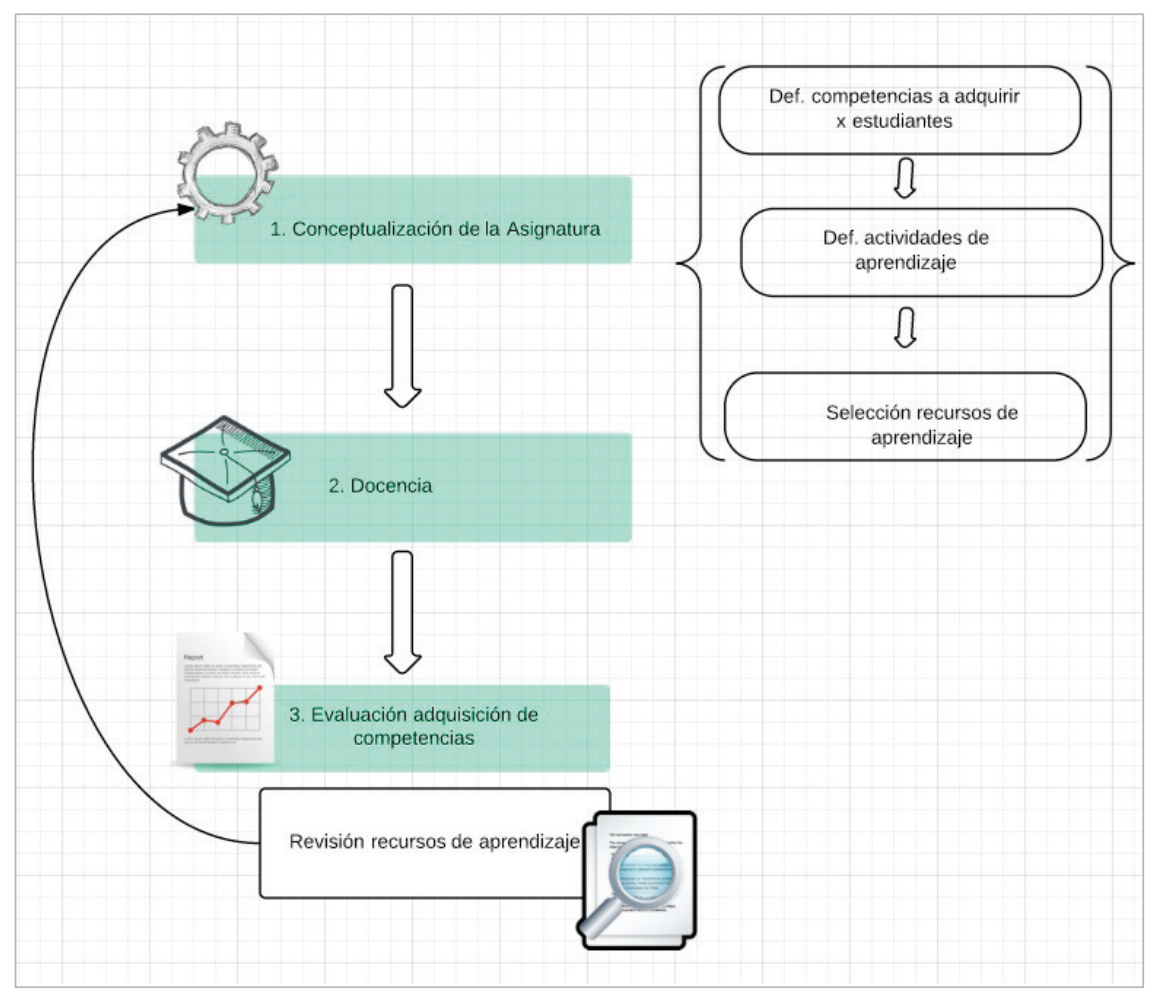

Figura 7. Proceso debía realizar para conseguir unos servicios bibliotecarios relevantes y que se convirtieran en referencia para los usuarios:

- Guiar, asesorar y apoyar a los docentes en la búsqueda de recursos de aprendizaje para las actividades de aprendizaje de sus asignaturas.

- Formarlos en la búsqueda, evaluación y uso de información, así como en el conocimiento de las herramientas existentes en la Universidad y en el mercado editorial.

- Informar y difundir las fuentes de información de suscripción y en abierto disponibles en la biblioteca y en el mercado editorial que cubren sus intereses temáticos.

- Ayudar a difundir la actividad docente de la Universidad.

Los recursos de aprendizaje comprenden los contenidos, los espacios y las herramientas necesarios para llevar a cabo las actividades de aprendizaje y su evaluación

\subsection{Definición de los servicios}

Los servicios dirigidos a los docentes que se ofrecen desde la BVUOC se inician cuando la Universidad toma la decisión de abrir un nuevo programa. En ese momento, el equipo de SBA envía a los profesores una primera selección de recursos y herramientas disponibles en la Biblioteca que pueden considerarse de interés y utilidad para la docencia y posterior actividad de aprendizaje por parte de los estudiantes.

En la UOC, la definición de las asignaturas tiene lugar en el momento de diseñar los programas. Se determinan las competencias que los estudiantes deberán adquirir, se crean las actividades de aprendizaje que permitirán la adquisición de dichas competencias y se seleccionan los recursos de aprendizaje que guiarán y proporcionarán a los estudiantes el apoyo necesario para el seguimiento de la asignatura.

Una vez la asignatura ha sido conceptualizada, llega el momento de ofrecerla para que los estudiantes se matriculen e inicien el proceso de docencia y aprendizaje. Durante esta etapa, profesores y estudiantes podrán comprobar si tanto las actividades como los recursos de aprendizaje asociados se adecúan a la adquisición de las competencias definidas en la asignatura.

Finalizado el período de docencia, se inicia un proceso de reflexión y análisis que permite evaluar al detalle la evolución de la asignatura, la utilidad de los recursos de aprendizaje, etc., así como detectar mejoras para implementar en el siguiente semestre o curso. 
Tabla 2. Carta de servicios

\begin{tabular}{|c|c|}
\hline $\begin{array}{l}\text { Servicios para la docencia } \\
\text { (¿Qué necesitas?) }\end{array}$ & Definición / Descripción \\
\hline $\begin{array}{l}\text { Realizar un encargo de autoría y/o entregar originales } \\
\text { para editar }\end{array}$ & $\begin{array}{l}\text { Permite formalizar el encargo de autoría de un contenido a un autor y facilitar la entrega } \\
\text { de originales para su posterior edición y producción en forma de recursos de aprendizaje } \\
\text { UOC. }\end{array}$ \\
\hline $\begin{array}{l}\text { Reaprovechar materiales UOC ya existentes para mi } \\
\text { asignatura }\end{array}$ & $\begin{array}{l}\text { Permite formalizar la solicitud de uso de unos materiales ya existentes, en la asignatura } \\
\text { de la que el profesor es responsable. Su reaprovechamiento será posible siempre y cuan- } \\
\text { do el contrato de autoría así lo permita. }\end{array}$ \\
\hline $\begin{array}{l}\text { Consultar el estado del envío de materiales de mi } \\
\text { asignatura }\end{array}$ & $\begin{array}{l}\text { Permite consultar el estado del envío de los materiales de una asignatura en concreto. } \\
\text { Envío a autores, equipo docente y estudiantes, siempre que el recurso de aprendizaje sea } \\
\text { susceptible de tener asociado el envío obligatorio. }\end{array}$ \\
\hline $\begin{array}{l}\text { Solicitar una búsqueda y selección de recursos de } \\
\text { aprendizaje para mi asignatura (programa / estudios) }\end{array}$ & $\begin{array}{l}\text { Permite solicitar una búsqueda y selección de recursos sobre un tema y/o área de interés } \\
\text { de una asignatura, programa o estudio, basado en la colección de la Biblioteca y recursos } \\
\text { externos. }\end{array}$ \\
\hline $\begin{array}{l}\text { Solicitar y/o renovar los recursos externos de mi } \\
\text { asignatura }\end{array}$ & $\begin{array}{l}\text { Permite solicitar como recurso de aprendizaje de la asignatura cualquier tipo de recurso } \\
\text { interno UOC más allá de los materiales UOC [guías de estudio, PEC (prácticas de evalua- } \\
\text { ción continuada), soluciones, etc.] y externo (libro-manual, dispositivos, placas informá- } \\
\text { ticas, etc.), enlaces web, artículos de revista, capítulos y/o partes de libro, que provienen } \\
\text { de las fuentes de información suscritas de la Biblioteca y/o que pueden ofrecerse de } \\
\text { terceros en el aula, a través de la consiguiente cesión de derechos de autor. }\end{array}$ \\
\hline $\begin{array}{l}\text { Solicitar y/o modificar la bibliografía recomendada de } \\
\text { mi asignatura }\end{array}$ & $\begin{array}{l}\text { Permite solicitar la creación de la bibliografía recomendada de una asignatura nueva, y/o } \\
\text { modificar la bibliografía ya existente de las asignaturas. }\end{array}$ \\
\hline $\begin{array}{l}\text { Evaluar los recursos de aprendizaje de mi asignatura } \\
\text { (uso y coste) }\end{array}$ & $\begin{array}{l}\text { Permite solicitar el asesoramiento del bibliotecario especializado en relación con la } \\
\text { utilización de los recursos de aprendizaje de una asignatura, y el coste de los mismos, } \\
\text { con el objetivo de mejorar la satisfacción de los estudiantes y rentabilizar los recursos } \\
\text { económicos que la institución pone a disposición de los estudios. }\end{array}$ \\
\hline $\begin{array}{l}\text { Estar al día sobre un tema ámbito de conocimiento de } \\
\text { tu interés }\end{array}$ & $\begin{array}{l}\text { Relación de contenidos y herramientas de la Biblioteca que facilitan que el profesor pue- } \\
\text { da mantenerse fácilmente al día sobre un tema y/o un área de conocimiento. }\end{array}$ \\
\hline $\begin{array}{l}\text { Formación de la Biblioteca en las aulas virtuales de mi } \\
\text { asignatura }\end{array}$ & $\begin{array}{l}\text { Relación de herramientas de formación de la Biblioteca disponibles para el uso del equi- } \\
\text { po docente en el aula virtual. }\end{array}$ \\
\hline
\end{tabular}

A partir del contexto explicado anteriormente y como resultado de todo el análisis previo, en la tabla 2 se definen los servicios dirigidos a la docencia que el equipo de SBA ha desarrollado desde la perspectiva del usuario docente que accederá desde la web de la BVUOC.

\section{Resultado}

Se han obtenido algunos resultados preliminares desde la puesta en marcha del servicio de apoyo a la docencia, en virtud de: una serie de indicadores proporcionados por las aplicaciones internas de gestión de los recursos en las aulas, la retroalimentación de los bibliotecarios del equipo y de los profesores que han solicitado este servicio.

Se han considerado los siguientes indicadores de gestión de recursos de aprendizaje en las aulas de la UOC:

- número de peticiones de búsqueda de recursos solicitadas por el profesorado de la UOC y gestionadas por la BVUOC.

- número de recursos de aprendizaje publicados en las aulas durante el curso anterior (2013-2014) y posterior (2014-2015) a la puesta en marcha del servicio de apoyo a la docencia. Este indicador permite comprobar si ha habido un incremento de recursos.

El primer indicador, número total de peticiones recibidas y gestionadas entre los meses de junio y octubre (2014), es de 109. Estas solicitudes son de diversos tipos: búsquedas generales sobre el estado de la cuestión de un tema, con vistas a proponer un nuevo programa de estudios; búsquedas más específicas para poner en marcha nuevas asignaturas o rediseñar otras existentes, etc. Las peticiones proceden del profesorado de las áreas de conocimiento de la Universidad (253 profesores, según datos oficiales de la Generalitat de Catalunya a 31 de diciembre de 2013). Por tanto, se ha gestionado una media de 21,8 solicitudes mensuales que a su vez provienen de un $43 \%$ del profesorado de la Universidad.

Para el segundo indicador se han extraído datos del gestor documental de la BVUOC que permite registrar, asignar y publicar los recursos de aprendizaje (documentos electrónicos, artículos, capítulos de libro, enlaces web, actividades, etc.) en las aulas. La tabla 3 muestra los datos del curso actual en vigencia, 2014-2015 (septiembre de 2014-febrero de 2015) respecto al curso anterior 2013-2014, desglosado en dos semestres (septiembre de 2013-febrero de 2014 y febrero-junio de 2014). Los datos que se han tenido en cuenta son el número total de recursos de aprendizaje activos en las aulas, que se componen de:

- Recursos que han comportado alguna gestión (recursos de nueva incorporación y otros que han sido modificados -aquellos ya existentes que se actualizan, ya sea por un cambio en la licencia de uso, la modificación del url, etc.).

- Recursos ya existentes en las aulas, que se mantienen de cursos anteriores porque no han necesitado ningún tipo de gestión / actualización. 
Tabla 3. Indicadores

\begin{tabular}{|l|c|c|c|c|c|}
\hline & $\begin{array}{c}\text { Recursos de nueva } \\
\text { incorporación }\end{array}$ & $\begin{array}{c}\text { Recursos modifi- } \\
\text { cados }\end{array}$ & $\begin{array}{c}\text { Total de recursos } \\
\text { gestionados }\end{array}$ & $\begin{array}{c}\text { Recursos existentes (no } \\
\text { requieren gestión) }\end{array}$ & $\begin{array}{c}\text { Total de recursos } \\
\text { activos en las aulas }\end{array}$ \\
\hline $\begin{array}{l}20141 \\
\text { (sept. 14-feb. 15) }\end{array}$ & 953 & 792 & $\mathbf{1 . 7 4 5}$ & 9.782 & $\mathbf{1 1 . 5 2 7}$ \\
\hline $\begin{array}{l}20132 \\
\text { (feb.-jun. 14) }\end{array}$ & 714 & 471 & $\mathbf{1 . 1 8 5}$ & 9.319 & $\mathbf{1 0 . 5 0 4}$ \\
\hline $\begin{array}{l}20131 \\
\text { (sept. 13-feb. 14) }\end{array}$ & 802 & 657 & $\mathbf{1 . 4 5 9}$ & 9.025 & $\mathbf{1 0 . 4 8 4}$ \\
\hline
\end{tabular}

Se observa que en lo que llevamos del curso actual, 20142015, se han contabilizado 11.527 recursos en las aulas de la UOC. Si comparamos este dato con el número total de recursos registrados en los dos semestres anteriores del curso 2013-2014, observamos un incremento de 1.043 respecto al primer semestre (septiembre de 2013-febrero de 2014) y de 1.023 respecto al segundo (febrero-junio de 2014). Por consiguiente, en el curso actual se ha producido un claro aumento en el número de recursos activos en las aulas. Este hecho podría considerarse como un éxito si además tenemos en cuenta que el total puede crecer más hasta la finalización del semestre (febrero de 2015).

\section{Se ha percibido un mayor interés de los docentes por la Biblioteca}

Si nos fijamos en el detalle de los recursos que han requerido de alguna gestión por parte del equipo SBA, se comprueba un incremento de 286 recursos en el primer semestre de 2013-2014 y de 560 en el segundo. Esto significa un aumento en la gestión de recursos en las aulas durante el curso actual respecto al anterior, es decir, se han recibido y gestionado más solicitudes del profesorado para incorporar o actualizar recursos de aprendizaje en las aulas.

En cuanto a la opinión de equipo de SBA, hay una sensación generalizada de que el nuevo servicio de apoyo a la docencia ha propiciado una mayor conexión y colaboración con el profesorado; un acercamiento de cada bibliotecario temático con los profesores de su área de conocimiento/estudios. También se ha percibido un mayor interés de los docentes por la Biblioteca, por lo que se desprende de las respuestas de la encuesta y el aumento de solicitudes de búsqueda y publicación de recursos de aprendizaje en las aulas.

Respecto a las reacciones de los profesores que han participado en el nuevo servicio de apoyo a la docencia, en general, se manifiesta la satisfacción con el trabajo realizado. Algunos comentan que les ha servido para "saber que existe un mundo de posibilidades que podemos explorar"; otros especifican que las búsquedas de recursos de aprendizaje les han servido para "preparar la docencia" de sus asignaturas, "a título informativo del estado de la cuestión sobre la temática", "para añadir la referencia de un libro y de un artículo como fuentes de información en las aulas" o para "utilizar algún recurso en una de las actividades programadas".

Todas estas reacciones conducen a una idea de retroalimentación, si consideramos que la salida de información de un sistema o su influencia (a través del servicio de apoyo a la docencia) repercute en la introducción de nueva información (utilización de recursos en las aulas) en el mismo. Esta retroalimentación sería positiva si continúa produciendo el mantenimiento o incremento del sistema existente.

\section{Conclusiones}

El proceso de definición de una carta de servicios específica para los docentes nos ha obligado a llevar a cabo una serie de acciones previas tales como un análisis interno DAFO, un benchmarking de servicios dirigidos a los docentes en otras universidades nacionales e internacionales, y la recogida de más de un centenar de respuestas de profesores de la UOC mediante una encuesta. Consideramos que todas estas acciones ya son de por sí consecuencias muy positivas del proceso llevado a cabo. El resultado de las mismas ha ayudado a definir los servicios que ofrecemos a nuestros usuarios, a medida de sus necesidades, y también a orientar nuestra actividad de manera más eficiente.

Se ha conseguido que todas las áreas de conocimiento de la UOC estén representadas en los resultados de la encuesta. No obstante, cabe destacar que la baja participación del profesorado de las áreas de derecho y ciencia política, ciencias de la salud y Escuela de doctorado podría mejorarse mediante el contacto directo por parte del bibliotecario de cada área de conocimiento a través de entrevistas personales y propuestas de soluciones a medida.

\section{Se constata un aumento en la gestión y en el número de recursos de aprendiza- je disponibles en las aulas debido a una mayor demanda y uso de la biblioteca}

Se constata un aumento en la gestión y en el número de recursos de aprendizaje disponibles en las aulas del curso actual respecto al anterior debido a una mayor demanda y uso de la biblioteca y de su servicio de apoyo a la docencia por parte del profesorado. Por otro lado, se evidencia una mejora de los recursos de aprendizaje que se ofrecen a los estudiantes en cuanto a actualización, idoneidad, diversidad, etc.

Se ha producido una tendencia, tal vez incipiente, hacia la sostenibilidad de los recursos de aprendizaje, ya que muchas gestiones han consistido en la reutilización de materiales ya existentes y en la utilización de recursos disponibles 
en la colección digital de la biblioteca. Este último hecho probablemente sea debido a la mayor intervención de los bibliotecarios en el proceso de preparación de la docencia, es decir, en el asesoramiento más personalizado fruto del proceso de acompañamiento al profesor en el diseño de su asignatura, que es el principal objetivo del liaison librarian.

Por último, la carta de servicios específica para docentes resultante es tan sólo un punto de partida. Dentro del plan de mejora continua de la BVUOC se establece una evaluación anual que permite la evolución y adecuación de los servicios bibliotecarios a las necesidades de los usuarios.

\section{Bibliografía}

ACRL Research Planning and Review Committee (2014). "Top trends in academic libraries: A review of the trends and issues affecting academic libraries in higher education". College \& research libraries news, v. 75, n. 6, pp. 294-302. http://crln.acrl.org/content/75/6/294.full

Alam, Sher-Malik (2011). "Changing roles of teachers". New straits times, 4. pp. 4-6.

Cervera, Albert (2010). "Document management in the Open University of Catalunya (UOC) classrooms". D-lib magazine, July/August, v. 16, n. 7/8.

http://www.dlib.org/dlib/july10/cervera/07cervera.html http://dx.doi.org/10.1045/july2010-cervera

Connaway, Lynn-Silipigni; Faniel, Ixchel (2014). Reordering Ranganathan: Shifting user behaviors, shifting priorities. Dublin, OH: OCLC Research. ISBN: 9781556534737

http://www.oclc.org/research/publications/library/2014/ oclcresearch-reordering-ranganathan-2014-overview.html

Domínguez-Aroca, María-Isabel (2005). “La biblioteca universitaria ante el nuevo modelo de aprendizaje: docentes y bibliotecarios, aprendamos juntos porque trabajamos juntos". RED. Revista de educación a distancia, no monográfico II.

http://www.um.es/ead/red/M4/dominguez9.pdf

Extremeño, Ana; Amante, Maria-João; Da-Costa, AntónioFirmino (2013). "La universidad del siglo XXI: nueva docencia, nuevo bibliotecario". Revista española de documentación científica, v. 36, n. 2, pp. 1-7.

http://redc.revistas.csic.es/index.php/redc/article/ view/794/935

Gillaspy-Steinhilper, Andrea (2012). “I don't have time to teach that: The benefits of faculty-librarian collaborations". Faculty focus, July 16. http://www.facultyfocus.com/articles/instructional-design/ i-dont-have-time-to-teach-that-the-benefits-of-facultylibrarian-collaborations

Henry, Jo (2014). "Academic library liaison programs: four case studies". Library review, v. 61, n. 7, pp. 485-496. http://dx.doi.org/10.1108/00242531211288236

Holbrook, Allyson (1984). "The subject librarian and social scientists: liaison in a university setting". Aslib proceedings, v. 36, n. 6 , pp. $269-275$

http://dx.doi.org/10.1108/eb050932

Johnson, Larry; Adams-Becker, Samantha; Estrada, Victoria; Freeman, Alex (2014). NMC horizon report: 2014 library edition. Austin, Texas: The New Media Consortium. ISBN: 9780991482887

http://cdn.nmc.org/media/2014-nmc-horizon-reportlibrary-EN.pdf

Lippincott, Joan; Vedantham, Anu; Duckett, Kim (2014). "Libraries as enablers of pedagogical and curricular change". Educause review online.

http://www.educause.edu/ero/article/libraries-enablerspedagogical-and-curricular-change

Ranganathan, Shiyali R. (1931). The five laws of library science. Madras: The Madras Library Association. http://hdl.handle.net/2027/uc1.\$b99721

Santos-Hermosa, Gema (2012). "Faculty-librarians collaboration in an e-learning experience: resources management and training in Open University of Catalonia (UOC)". En: 2012 teaching \& learning symposium. http://hdl.handle.net/10609/18482

Sigalés, Carles (2013). “¿Qué estamos haciendo las universidades para adaptar la docencia a las innovaciones de la tecnología?". En: Las nuevas formas de enseñanza en la Universidad digital. Seminario bienal La universidad digital. http://catedraunesco.es/seminario/2013-2015/jornada2/ Presentacion-CSigales.pdf

Smith, Risë L.; Mundt, Karl E. (2014). Philosophical shift: Teach the faculty to teach information literacy. Association of College \& Research Libraries.

http://www.ala.org/acrl/publications/whitepapers/ nashville/smith

Universitat Oberta de Catalunya (2014). Pla estratègic 20142020.

http://www.uoc.edu/portal/ca/estrategia/documentacio/20131010_ Sessio_Informativa_UOC_Pla_estrategic.pdf 
